\title{
émulations
}

\section{Célia Gissinger-Bosse - Être juré populaire en cour d'assises}

Vincent Jacquet

Émulations - Revue de sciences sociales

2018. Comptes rendus critiques. En ligne

\section{Article disponible à l'adresse suivante}

https://ojs.uclouvain.be/index.php/emulations/article/view/7513

\section{Pour citer cet article}

Vincent Jacquet, « Célia Gissinger-Bosse - Être juré populaire en cour d'assises », Émulations, en ligne. Mise en ligne le 19 novembre 2018.

DOI : 10.14428/emulations.cr.050

Distribution électronique : Université catholique de Louvain (Belgique) : ojs.uclouvain.be

(C) Cet article est mis à disposition selon les termes de la Licence Creative Commons Attribution, Pas d'Utilisation Commerciale 4.0 International. http://creativecommons.org/licenses/by-nc/4.0/

Éditeur : Émulations - Revue de sciences sociales / Presses universitaires de Louvain https://ojs.uclouvain.be/index.php/emulations

ISSN électronique : $1784-5734$

UCL PRESSES

UNIVERSITAIRES

DE LOUVAIN 


\title{
Célia Gissinger-Bosse - Être juré populaire en courd'assises
}

\begin{abstract}
Vincent Jacquet $^{1}$
Recensé : Célia Gissinger-Bosse (2017), Être juré populaire en cour d'assises, Paris, Éditions de la Maison des sciences de l'homme (« Le (bien) commun »), 248 p.

Devenir, être et avoir été juré d'assises. C'est ce triple objet d'étude que Célia GissingerBosse se propose d'examiner dans cet ouvrage issu de sa thèse de doctorat en sciences de l'information et de la communication. Elle invite à suivre au plus près le parcours de citoyens amenés à exercer cette fonction singulière. Le grand public est relativement familier de l'institution du jury d'assises, approchée par les séries américaines et le traitement médiatique de quelques affaires criminelles retentissantes. Mais une fois situés au cœur d'un procès, juste à côté des juges professionnels, les citoyens sont amenés à découvrir un espace qui leur est pour la plupart inconnu, ainsi qu'à exercer une faculté très particulière : celle de juger. En mobilisant des observations de procès et des entretiens semi-directifs avec des citoyens qui ont siégé comme jurés, l'auteure retrace avec minutie les différentes étapes de cette expérience.

L'ouvrage est structuré en trois grandes parties selon une logique chronologique, allant de la convocation officielle au retour à la vie de tous les jours après le procès. Dans la première partie, l'auteure s'attache à décrire le processus par lequel les citoyens que le sort a sélectionnés deviennent des jurés populaires. Ceci implique un certain détachement - tant physique que symbolique - de leur quotidien pour prendre place au sein d'un espace social qui leur est étranger. Il s'agit de découvrir le déroulé de la procédure, le rôle des différents acteurs, les différents registres de communication, ainsi que la place qui leur est accordée dans cet espace marqué par de nombreuses règles formelles et informelles. Cette socialisation se fait à travers différents rites, comme celui du tirage au sort des jurés et de la prestation de serment. Ces rites participent à matérialiser le passage au statut de juré. Les extraits mobilisés montrent également les doutes et les interrogations présentes dans le chef des citoyens quant à leur propre légitimité à siéger au cœur de ces cours d'assises.

L'expérience spécifique du jugement fait l'objet de la deuxième partie de l'ouvrage. Célia Gissinger-Bosse y compare celle-ci à un voyage initiatique où le juré, face au collectif et à lui-même, doit trouver les traces d'une intime conviction dans un labyrinthe complexe. Les citoyens font l'expérience de l'altérité en découvrant la procédure judiciaire, mais également le contexte dans lequel fut perpétré le crime qu'ils ont à connaitre. Ce faisant, les jurés relatent
\end{abstract}

${ }^{1}$ Chargé de recherches (F.R.S.-FNRS) en science politique à I'Université catholique de Louvain. 
l'effort d'empathie qu'ils ont réalisé afin d'aller au-delà de leur point de vue personnel pour prendre en considération une affaire dans ses multiples perspectives. Cette multiplicité est incarnée par le débat contradictoire entre les parties qui ne cesse de mettre en scène la complexité des faits. Aux yeux des personnes interrogées, c'est ce qui fait la valeur de leur jugement final. Le mouvement destiné à se départir « d'un jugement à l'emporte-pièce » (p. 101) est essentiel. Il vient nourrir la recherche de l'intime conviction à laquelle l'auteure consacre un long développement en puisant dans les travaux de Kant et Arendt (notamment Arendt, 1991 et 1995 ; Kant, 2015 [1790]) comme dans l'interprétation qu'en font les jurés. Elle conclut que les jurés populaires « font l'expérience de l'intersubjectivité, de l'empathie et du sentir en commun » (p. 16), reconsidérant par-là l'opposition simpliste entre émotion et raison.

La troisième partie de l'ouvrage s'attache à étudier plus précisément les changements induits par une telle expérience. La thèse centrale défendue par l'auteure consiste à considérer cette évolution comme une conversion démocratique. L'expérience du jury populaire change les individus qui y prennent part. Les jurés expliquent en effet qu'ils ont découvert une attirance pour la chose publique, une nouvelle vision de leur rôle dans la cité. Ils ont l'impression d'être (pour la première fois) reconnus comme des citoyens. Ceci passe par une transformation de leur représentation de la justice qui devient plus horizontale. Ils témoignent également d'une rupture par rapport à leur volonté initiale de vengeance qui se dissipe peu à peu au cours du procès, découvrant par là une capacité à prendre de la distance avec leur propre colère. De la sorte, les jurés se découvrent des compétences démocratiques qu'ils ne soupçonnaient pas (apprentissage de la neutralité, prise de parole et argumentation).

Le caractère novateur et passionnant de l'ouvrage tient pour beaucoup au type d'approche mobilisé. Le manuscrit est nourri par de très nombreux extraits d'entretiens qui illustrent les propos de nature plus abstraite. De la sorte, il est possible de saisir, voire de ressentir, les différentes phases par lesquelles passent les personnes qui sont tirées au sort. Ceci vient contrebalancer les travaux classiques sur le jury, principalement nord-américains, qui analysent de façon extériorisante l'influence de la composition du jury en termes de caractéristiques sociodémographiques sur l'issue du procès (Devine et al., 2001; Sommers, 2006). L'ouvrage prolonge en fait des travaux plus récents qui mettent au jour les perceptions des jurés lors des audiences et des délibérations (Jolivet, 2006; Matthews et al., 2004 ; Sprain et Gastil, 2013 ; Jellab et Giglio-Jacquemot, 2012). L'originalité du propos consiste à montrer qu'au-delà des multiples épreuves que traversent les jurés, ceux-ci expérimentent fondamentalement un processus de changement démocratique.

En outre, Célia Gissinger-Bosse parvient à rendre compte, de façon remarquable, de la réflexivité des acteurs. L'ouvrage fournit en effet une description du parcours complexe des jurés qui s'interrogent continuellement sur leur propre comportement et sur ce qui est attendu d'eux. C'est par exemple le cas lorsqu'elle fait état des moments où les jurés cherchent à se rassurer dans leur discussion avec leurs pairs sur les éléments de culpabilité. La complexité humaine est donc prise au sérieux, tout comme les multiples tensions contradictoires qui peuvent traverser les individus participant à cette expérience singulière. Ce travail est en ce sens 
particulièrement important pour l'étude de la participation des citoyens au sein des cours d'assises, mais aussi, plus largement, dans la sphère publique démocratique.

Certaines limites de l'étude invitent, bien entendu, à développer d'autres recherches. Notamment, la description chronologique comporte certaines zones d'ombre. L'ouvrage décrit en détail comment les jurés ressentent cette expérience de façon chronologique. Mais il n'est quasiment pas fait état de différences entre les personnes interrogées. L'écriture donne l'impression que tous les jurés passent exactement par les mêmes phases. Ce résultat soulève certaines questions. En comparant de façon systématique les entretiens entre eux, observe-ton une telle similarité dans le discours des jurés ? Si tel est le cas, cette homogénéité pourrait être interrogée. Le propre du jury populaire et du tirage au sort qui lui est associé consiste à rassembler des personnes aux socialisations et parcours de vie très différents. Comment expliquer que, malgré cette disparité, tous conçoivent et vivent leur rôle de juré de la même façon ? Également, l'auteure ne semble pas souligner les disparités entre les différents procès observés et pourtant situés dans des contextes nationaux différents (France et Belgique). Cette relative ressemblance pose question face à des pays aux cultures politiques différentes. Enfin, l'auteure précise dans l'annexe méthodologique que certains entretiens ont été menés directement après les procès, alors que d'autres bien des années plus tard. Les conclusions présentées ne font pas état de différences d'appréhension du procès au fil du temps. Ceci signifie-t-il que le changement démocratique opéré n'évolue pas progressivement ? Ces quelques questions invitent à poursuivre le travail réalisé par Célia Gissinger-Bosse.

\section{Bibliographie}

ARENDT H. (1991), Juger : sur la philosophie politique de Kant, Paris, Éditions du Seuil (« Libre examen. Politique »).

ARENDT H. (1995), Qu'est-ce que la politique?, Paris, Éditions du Seuil.

Devine D. J., Clayton L. D., Dunford B. B., Seying R. et Pryce J. (2001), « Jury decision making: 45 years of empirical research on deliberating groups ", Psychology, public policy, and law, vol. $7, n^{\circ} 3$, p. $622-727$.

Jellab A., Giglo-Jacquemot A. (2012), «Des profanes en justice. Les jurés d'assises, entre légitimité et contestation du pouvoir des juges », Politix, vol. 97, $\mathrm{n}^{\circ} 1$, p. 149-176.

Jolivet A. (2006), «Juré en cour d'assises : découverte d'un monde social et expérience de sociabilité au sein d'un groupe restreint », Droit et société, vol. 62, n 1, p. 203-222.

KANT E. (2015 [1790]), Critique de la faculté de juger, Paris, Flammarion.

MATtheWs R., HANCOCK L., BRIGGS D. (2004), Jurors' perceptions, understanding, confidence and satisfaction in the jury system: a study in six courts, Londres, Home office. 
SOMmerS S. R. (2006), " On racial diversity and group decision making: identifying multiple effects of racial composition on jury deliberations", Journal of personality and social psychology, vol. 90, $\mathrm{n}^{\circ} 4$, p. 597-612.

SPRAIN L., GASTIL J. (2013), «What Does It Mean to Deliberate? An Interpretative Account of Jurors' Expressed Deliberative Rules and Premises ", Communication Quarterly, vol. 61, $n^{\circ} 2$, p. 151-171. 